we should wear becomes the more pressing question.

Holowka and colleagues argue that thick calluses preserve sensitivity because their hardness enables mechanical stimuli from the ground to be transmitted, with little dampening, to deep layers of the skin in which the key mechanoreceptors are located. If so, shoes with hard soles should be predicted to do the same job as calluses. Indeed, the hard-soled shoes used by drivers competing in Formula 1 races provide even greater than normal sensitivity at high frequencies of vibration ${ }^{16}$.

More research will be needed to fully understand the effect of shoe soles on gait. Humans are not like machines, in which just one variable at a time can be studied. Human movement is a complex, dynamic system, and changing even one variable, such as shoe-sole stiffness, will probably trigger other physiological and behavioural changes. For example, running when using cushioned soles, compared with running barefoot, triggers changes in how the foot makes contact with the ground (called the strike pattern $)^{17}$, and also causes the arch of the foot to behave more stiffly ${ }^{18}$.

Holowka et al. conducted an experiment using a treadmill apparatus to quantify impact forces, which are the forces that the foot encounters immediately after it strikes the ground. They found that even if uncushioned shoes were used to mimic a callus-like sole, these shoes did not exactly mirror the effect of calluses during foot strike. Compared with their observations of unshod individuals, such footwear led to a slower rise in the impact force and a higher impulse (the product of the force and duration of the impact phase, which is when the foot hits the ground and slows abruptly).

It makes sense that preserving foot sensitivity is useful, especially if maintaining stability is challenging. This is true for gymnasts and also for older people, in whom faculties such as vision, balance and foot sensitivity decline naturally with age. Shoes with hard soles might therefore be a good idea for such individuals. Indeed, wearing hard-soled shoes can reduce the risk of older people falling ${ }^{19}$. Holowka and colleagues' work helps to explain why this is so. Although this mystery has been solved, much remains to be discovered about what affects how humans walk..

Kristiaan D'Août is in the Department of Musculoskeletal Biology, University of Liverpool, Liverpool L7 8TX, UK. e-mail:kristiaan.daout@liverpool.ac.uk

1. Senut, B. et al. C. R. Acad. Sci. IIA 332, 137-144 (2001).

2. Brunet, M. et al. Nature 418, 145-151 (2002).

3. McDougall, I., Brown, F. H. \& Fleagle, J. G. Nature 433, 733-736 (2005).

4. Trinkaus, E. \& Shang, H. J. Archaeol. Sci. 35, 1928-1933 (2008).

5. Holowka, N. B. et al. Nature 571, 261-264 (2019).

6. Alam, U. et al. Diabetes Ther. 8, 1253-1264 (2017).

7. Höhne, A., Ali, S., Stark, C. \& Brüggemann, G.-P.
Eur. J. Appl. Physiol. 112, 3829-3838 (2012).

8. Alfuth, M. \& Rosenbaum, D. Footwear Sci. 4, 1-22 (2012).

9. Shulman, S. B. J. Natl Assoc. Chiropodists 49, 26-30 (1949).

10.Sim-Fook, L. \& Hodgson, A. J. Bone Joint Surg. Am. 40, 1058-1062 (1958)

11.D'Août, K., Pataky, T. C., De Clercq, D. \& Aerts, P. Footwear Sci. 1, 81-94 (2009).

12. Willems, C., Stassijns, G., Cornelis, W. \& D'Août, K. Am. J. Phys. Anthropol. 162, 782-793 (2017).

13.Engle, E. T. \& Morton, D. J. J. Bone Joint Surg. 13, 311-318 (1931)

14.Bus, S. A. et al. Diabetes/Metab. Res. Rev.
32 (suppl.), 99-118 (2016).

15. Hoogkamer, W. et al. Sports Med. 48, 1009-1019 (2018).

16.Schlee, G., Sterzing, T. \& Milani, T. L. Eur. J. Appl. Physiol. 106, 305-309 (2009).

17.De Wit, B., De Clercq, D. \& Aerts, P. J. Biomech.33, 269-278 (2000).

18. Kelly, L. A., Lichtwark, G. A., Farris, D. J. \& Cresswell, A. J. R. Soc. Interface 13, 20160174 (2016).

19.Aboutorabi, A. et al. Prosthet. Orthot. Int. 40, 170-181 (2016).

This article was published online on 26 June 2019.

\title{
Low retention of impact material by the Moon
}

Simulations demonstrate that the Moon's ability to retain material from striking impactors is lower than was previously assumed. This finding helps to explain the scarcity of precious metals in the Moon relative to Earth. SEE LETTER P.226

\section{JAMES M. D. DAY}

A s a planetary body forms, precious metals, such as gold and iridium, are stripped from its rocky mantle and passed into its metallic core. Successive impacts with other objects then re-enrich the mantle in these elements - a process known as late accretion ${ }^{1,2}$. Measurements of lunar rocks show that the Moon is greatly depleted in precious metals compared with Earth ${ }^{3,4}$. This deficit implies that the ratio of mass added to Earth during late accretion compared with that added to the Moon is more than 1,000:1, which is substantially different from the predicted ratio ${ }^{5}$ of about 20:1. On page 226, Zhu et al. ${ }^{6}$ show that inefficient delivery of material from glancing impacts, combined with an early hot, molten stage on the Moon, can explain this anomalous input-mass ratio.

An analysis of the mass and composition ${ }^{7}$ of material added to a planetary body can be used to examine the body's formation. Moreover, late accretion is linked to the delivery of water and other volatile elements to Earth ${ }^{8}$, and such additions are probably a key factor in our planet's habitability. The low abundances of precious metals in lunar rocks ${ }^{3,4,9}$ has prompted the proposal of competing models to explain the anomalous input-mass ratio between Earth and the Moon.

At one extreme, these models include delivery of material by a few massive impactors (larger than 2,500 kilometres in diameter) that preferentially struck Earth ${ }^{5}$. At the other, focusing of small objects (less than $10 \mathrm{~m}$ in diameter) on to Earth might have produced similar effects ${ }^{10}$. It has

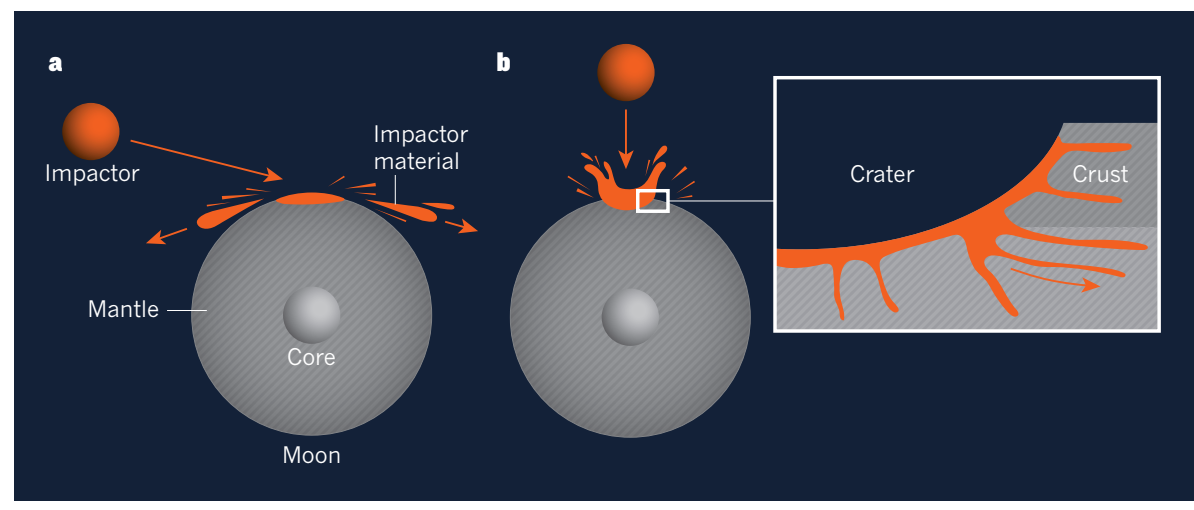

Figure 1 | Lunar glancing blows and direct hits. a, Zhu et al. ${ }^{6}$ show that the Moon's ability to retain material from an impactor depends on the angle of the impact with respect to the lunar surface. Lowangle impacts (glancing blows) can result in $80 \%$ of the impactor material being lost. The Moon's rocky mantle and metallic core are shown. b, By contrast, high-angle impacts (direct hits) lead to a substantial fraction of the impactor material being retained. After the impact (see inset), a crater forms, and the impactor material can be incorporated into both the lunar crust and mantle. 
also been suggested that the difference in precious-metal abundances between Earth and the Moon was caused by a drop in the flux of impacts during the period between 4.5 billion and 4.1 billion years ago ${ }^{11}$, just after the Solar System formed. These models generally assume that the Moon retained about half of the mass that was transferred to it by impactors.

Using millions of computational impact simulations, Zhu and colleagues examined the fraction of impactor mass that could be retained by planetary bodies. The authors simulated impacts at different velocities (10-20 km per second), and at low angles $\left(20^{\circ}\right)$ to high angles $\left(80^{\circ}\right)$ with respect to the body's surface (Fig. 1). They found that material from larger impactors is less effectively retained than that from smaller counterparts, and that highangle impacts deliver a larger mass fraction to the body than do low-angle impacts.

In the case of Earth, these results imply that the retention of impactor mass is generally high for all but the most glancing impacts with the most massive objects. For the Moon, which has a mass only about $1 \%$ of that of Earth, the shallower the angle of impact, and the more massive the impactor, the greater the likelihood that material would be lost, never reaching the Moon's surface or passing into its interior. Using crater diameters ${ }^{12}$ to establish the frequency and size of impactors striking the Moon, Zhu et al. discovered that impactormass retention probably changed modestly over time, and that the average retention was about $20 \%$, which is around three times lower than previous estimates.

Inefficient retention of material from objects striking the Moon partially offsets the difference between the theoretically and geochemically determined Earth-Moon input-mass ratios. Zhu and colleagues then argue that about $50 \%$ of late-accretion input mass was lost to the Moon's deep interior or core before 4.35 billion years ago, and that this loss explains any remaining discrepancy. Later, once the Moon had cooled, late-accretion input mass was distributed into the lunar mantle and crust. The authors further suggest that as many as 300 impact craters of more than $300 \mathrm{~km}$ in diameter might have existed on the Moon, but that fewer than 30\% of these craters are preserved today owing to impactderived erosion or to gradual subsidence (viscous relaxation) of the earliest craters in the hot lunar crust.

The suggestion of inefficient mass retention from glancing impacts negates the requirement for the proposed temporally varying impact fluxes ${ }^{11}$. However, the idea that precious metals were lost to the Moon's deep interior or core before 4.35 billion years ago is more problematic. Without evidence from craters for the impact flux to the Moon at that time, geochemistry is the only valid test of this idea. Loss of precious metals to a metallic core can lead to these elements being separated (fractionated) from one another ${ }^{13}$.
However, this chemical effect has not so far been detected in rocks from the lunar interior $^{3,4}$. Furthermore, low precious-metal abundances estimated for the lunar mantle make it difficult to envisage how such fractionation signatures could have been erased by further late accretion.

Zhu et al. also assume that the penetration of impactors through the lunar crust, which is about $40 \mathrm{~km}$ thick $^{14}$, would lead to all retained impactor material entering the mantle. But, in reality, this material would pollute both the crust and the mantle. Finally, because only a relatively small number of lunar rocks have been analysed, models such as the authors' that can reproduce precious-metal abundances in the Moon through simulations have limited resolution.

Nevertheless, the new models will be of value in understanding the evolution of planetary bodies, especially Mars. Current estimates for late-accretion input mass expressed as a function of total body mass are $0.02 \%$, about $0.5 \%$ and up to $0.7 \%$ for the Moon ${ }^{4}$, Earth $^{2}$ and $\mathrm{Mars}^{15}$, respectively. The estimate for Mars has been explained by early formation of the planet relative to Earth and the Moon, in addition to a constant input-mass flux in the Solar System's first 50 million to 100 million years ${ }^{15}$, and impacts involving massive objects ${ }^{5,16}$.

Extrapolation of Zhu and colleagues' models would suggest that Mars, which has a mass only about $11 \%$ of that of Earth, retains less material from large impactors than does Earth. Assuming that the two bodies were subjected to a similar number of glancing impacts, these models would imply that Mars had a proportionally greater late-accretion flux than did Earth. The combination of geochemistry and formation models will undoubtedly continue to improve our understanding of how Earth and its nearest neighbours came to be.

James M. D. Day is at the Scripps Institution of Oceanography, University of California, San Diego, La Jolla, California 92093, USA. e-mail:jmdday@ucsd.edu

1. Chou, C.-L. Proc. 9th Lunar Planet. Sci. Conf. 1 219-230 (1978); go.nature.com/2xn55sa

2. Walker, R. J. Chem Erde Geochem. 69, 101-125 (2009).

3. Day, J. M. D., Pearson, D. G. \& Taylor, L. A. Science 315, 217-219 (2007)

4. Day, J. M. D. \& Walker, R. J. Earth Planet. Sci. Lett. 423, 114-124 (2015)

5. Bottke, W. F., Walker, R. J., Day, J. M. D., Nesvorny, D. \& Elkins-Tanton, L. Science 330, 1527-1530 (2010).

6. Zhu, M.-H. et al. Nature 571, 226-229 (2019).

7. Fischer-Gödde, M. \& Kleine, T. Nature 541, 525-527 (2017).

8. Albarède, F. Nature 461, 1227-1233 (2009).

9. Day, J. M. D., Walker, R. J., James, O. B. \& Puchtel, I. S. Earth Planet. Sci. Lett. 289, 595-605 (2010).

10.Schlichting, H. E., Warren, P. H. \& Yin, Q.-Z. Astrophys. J. 752, 8 (2012).

11.Morbidelli, A., Marchi, S., Bottke, W. F. \& Kring, D. A. Earth Planet. Sci. Lett. 355-356, 144-151 (2012).

12. Neukum. G., Ivanov, B. A. \& Hartmann, W. K. Space Sci. Rev. 96, 55-86 (2001).

13. Mann, U., Frost, D. J., Rubie, D. C., Becker, H. \& Audétat, A. Geochim. Cosmochim. Acta 84, 593-613 (2012).

14.Wieczorek, M. A. et al. Science 339, 671-675 (2013).

15. Tait, K. T. \& Day, J. M. D. Earth Planet. Sci. Lett. 494, 99-108 (2018).

16.Woo, J. M. Y., Genda, H., Brasser, R. \& Mojzsis, S. J. Icarus 333, 87-95 (2019).

\section{T cells inhibit neural stem cells in old brains}

Age-dependent changes in their environment can impair stem cells' function. The finding that $T$ cells infiltrate the brains of aged mice and cause dysfunction of neural stem cells reveals a potential therapeutic target. SEE ARTICLE P.205

\section{ALLISON M. BOND \& HONGJUN SONG}

$\int \mathrm{r}$ $n$ a healthy adult, tissue-specific stem cells replenish damaged tissue and sustain plasticity (the addition of new cells) in organs. In two regions of the adult brains of most mammals (the subventricular zone of the lateral ventricles and the dentate gyrus of the hippocampus), neural stem cells generate new neurons, which contribute to brain plasticity and cognition ${ }^{1}$. However, there is still debate over whether new neurons are commonly generated in the adult human brain. The proliferation of neural stem cells in mammals decreases with age, resulting in a reduction in the number of new neurons formed over time, and the mechanism underlying this change is poorly understood ${ }^{2}$. On page 205, Dulken et al. ${ }^{3}$ examined how changes in the microenvironment of neural stem cells in the brains of old mice affect stem-cell proliferation.

Stem cells in an old brain are dysfunctional and are less likely to divide than are young stem cells ${ }^{4}$. However, the intrinsic properties of neural stem cells remain stable - both young and old neural stem cells have a similar potential to differentiate and proliferate in vitro ${ }^{5}$. Stem cells are located in a specialized microenvironment called a niche, which consists of molecules and other cells that interact with 